\title{
Serum Des-gamma-carboxy Prothrombin for Diagnosis of Adult Primary Cancer in Liver
}

\author{
Youdi Li and Jiu Chen \\ Department of Traditional Chinese Medicine, the First Affiliated Hospital, College of Medicine, Zhejing University, Hangzhou, China
}

\begin{abstract}
Objective: To explore the diagnostic accuracy of serum des-gamma-carboxy prothrombin (DCP) in adult primary cancer in liver.

Study Design: Descriptive study.

Place and Duration of Study: Department of Traditional Chinese Medicine, the First Affiliated Hospital, College of Medicine, Zhejing University, Hangzhou, China, from June 2016 to March 2018.

Methodology: A retrospective study of 1,190 cases was undertaken and all the participants were divided into Group 1 (non-cancer patients) and Group 2 (primary cancer in liver patients). The records of DCP and alpha fetoprotein (AFP) levels were extracted and compared between Group 1 and Group 2. The sensitivity, specificity, and cutoff point of DCP in Group 2 and its different histological types were calculated. The receiver operating characteristic (ROC) curves were obtained, and the areas under the receiver operating characteristic curve (AUROC) were calculated and compared in different histological types.

Results: The DCP and AFP levels in Group 2 were markedly higher than Group $1(p<0.001)$. For primary cancer in liver, the sensitivity, specificity, cutoff value, and AUROC of DCP were $64.2 \%, 91 \%, 56.5 \mathrm{mAU} / \mathrm{mL}$, and 0.807 (95\% Cl: $0.783-$ 0.832 ), respectively. For hepatocellular carcinoma (HCC), the sensitivity, specificity, cutoff value, and AUROC of DCP were $69.3 \%, 92.2 \%, 60.5 \mathrm{mAU} / \mathrm{mL}$, and 0.846 (95\% Cl: $0.817-0.875)$, respectively. For intrahepatic cholangiocarcinoma (ICC), the sensitivity, specificity, cutoff value, and AUROC of DCP were $13.6 \%, 93.2 \%, 76.5 \mathrm{mAU} / \mathrm{mL}$, and 0.512 (95\% Cl: 0.439 0.586 ), respectively. For combined hepatocellular-cholangiocarcinoma ( $\mathrm{cHCC}-\mathrm{CC}$ ), the sensitivity, specificity, cutoff value, and AUROC of DCP were $66.7 \%, 81.5 \%, 40.5 \mathrm{mAU} / \mathrm{mL}$, and 0.737 (95\% Cl: 0.585-0.888), respectively.

Conclusion: DCP could be considered not only for surveillance and diagnosis of primary cancer in liver, but also for differential diagnosis of its different histological types.
\end{abstract}

Key Words: Des-gamma-carboxy prothrombin, Diagnosis, Hepatocellular carcinoma, Intrahepatic cholangiocarcinoma, Combined hepatocellular-cholangiocarcinoma.

How to cite this article: Li Y, Chen J. Serum des-gamma-carboxy prothrombin for diagnosis of adult primary cancer in liver. $J$ Coll Physicians Surg Pak 2019; 29(10):972-6.

\section{INTRODUCTION}

Primary cancer in liver, mainly comprises hepatocellular carcinoma (HCC, $80 \%$ of cases), intrahepatic cholangiocarcinoma (ICC, $15 \%$ of cases), combined hepatocellular-cholangiocarcinoma (cHCC-CC, $1.3 \%$ of cases), undifferentiated primary cancer in liver, and hepatoblastoma, is the fifth commonly diagnosed cancer, but ranks second among the leading causes of cancer mortality worldwide.1,2 Additionally, the mortality of patients with most malignancies has decreased over the last decade with improvements in technologies, but the mortality of primary cancer in liver is still increasing. ${ }^{3}$ One of the main reasons is that majority of cases are initially asymptomatic and detected at advanced stages, which precludes the use of curative surgical therapy. 4

Correspondence to: Dr. Jiu Chen, Department of Traditional

Chinese Medicine, the First Affiliated Hospital, College of

Medicine, Zhejing University, Hangzhou, China

E-mail: linyunju@zju.edu.cn

Received: November 02, 2018; Revised: March 08, 2019;

Accepted: April 11, 2019
For ICC and $\mathrm{CHCC}-\mathrm{CC}$, which are more aggressive cancers with poorer prognoses, 2,5 early diagnosis and surgical resection are considered the only curative therapeutic option. ${ }^{2}$ These characteristics make early identification extremely crucial.

Ultrasound (US) has been regarded as the first-line imaging modality for detection of focal liver lesions and is the only recommended measurement for the surveillance of HCCs. ${ }^{6}$ However, the overall sensitivity of US in detecting $\mathrm{HCC}$ is only $0.593 .{ }^{7}$ The interpretation of US is not only operator-dependent but also difficult in the setting of small or atypical lesions or in persons who are obese or have underlying cirrhosis. Additionally, both ICC and CHCC-CC have no specific or characteristic features in US imaging.8,9 Magnetic resonance imaging (MRI) and enhanced computed tomography (CT) are more sensitive than US, but considering the cost, radiation exposure, nephrotoxicity, allergic and hypersensitivity reactions, they are not suitable for surveillance. Moreover, thus far, no reliable serum marker has been universally recognised for primary cancer in liver.5,6 
Therefore, the identification of sensitive and specific serum markers to complement US for the surveillance and early diagnosis of primary cancer in liver are urgently required. Serum des-gamma-carboxy prothrombin (DCP), also known as prothrombin-induced by vitamin $\mathrm{K}$ absence-II (PIVKA-II), has been widely studied in the diagnosis of HCC in a series of clinical trials and recommended in the surveillance programme as per Japanese guidelines. However, there are still two issues that need further exploration. First, the sensitivity, specificity, and cutoff points in previous studies have been inconsistent and in some instances, even conflicting. 10 Second, the diagnostic accuracy of DCP in other histological types of primary cancer in liver such as ICC and CHCC-CC has been seldom studied.

The objective of this study was to compare the diagnostic accuracy of DCP in primary cancer in liver and its different histological types (i.e., HCC, ICC, and cHCC-CC).

\section{METHODOLOGY}

A total of 1,190 participants were retrospectively enrolled at the Hospital, from June 2016 to March 2018. All the participants were first divided into two groups: Group 1 (non-cancer patients) and Group 2 (primary cancer in liver patients). The diagnosis of primary cancer in liver was made in accordance with the standards of diagnosis and treatment of primary liver cancer (2011 Edition) issued by the Ministry. ${ }^{11}$ The study was approved by the Ethics Committees of the Hospital.

For Group 2, the inclusion criteria were shown as follows: All DCP data were obtained before commencing any treatment. The diagnosis of primary cancer in liver was based on histological examination or the interpretation of appropriate imaging characteristics, as defined by above-mentioned standards. All the participants were $>18$ years old. The exclusion criteria were liver transplantation, taking vitamin $\mathrm{K}$ or warfarin within two weeks of DCP measurement. Patients showed evidence of other kinds of malignancies besides primary cancer in liver, histological examination showing other kinds of pathological types except HCC, ICC, and cHCC-CC, such as sarcomatous degeneration and hepatoblastoma. Nodules not confirmed due to biopsy or surgery, and refusal patients treated before DCP measurement.

The patients in Group 2 were subdivided into two groups: primary cancer in liver diagnosed with histological examination and primary cancer in liver diagnosed with imaging characteristics (without histological examination). The primary cancer in liver diagnosed with histological examination group was then subdivided again into three groups of HCC; ICC and CHCC-CC.

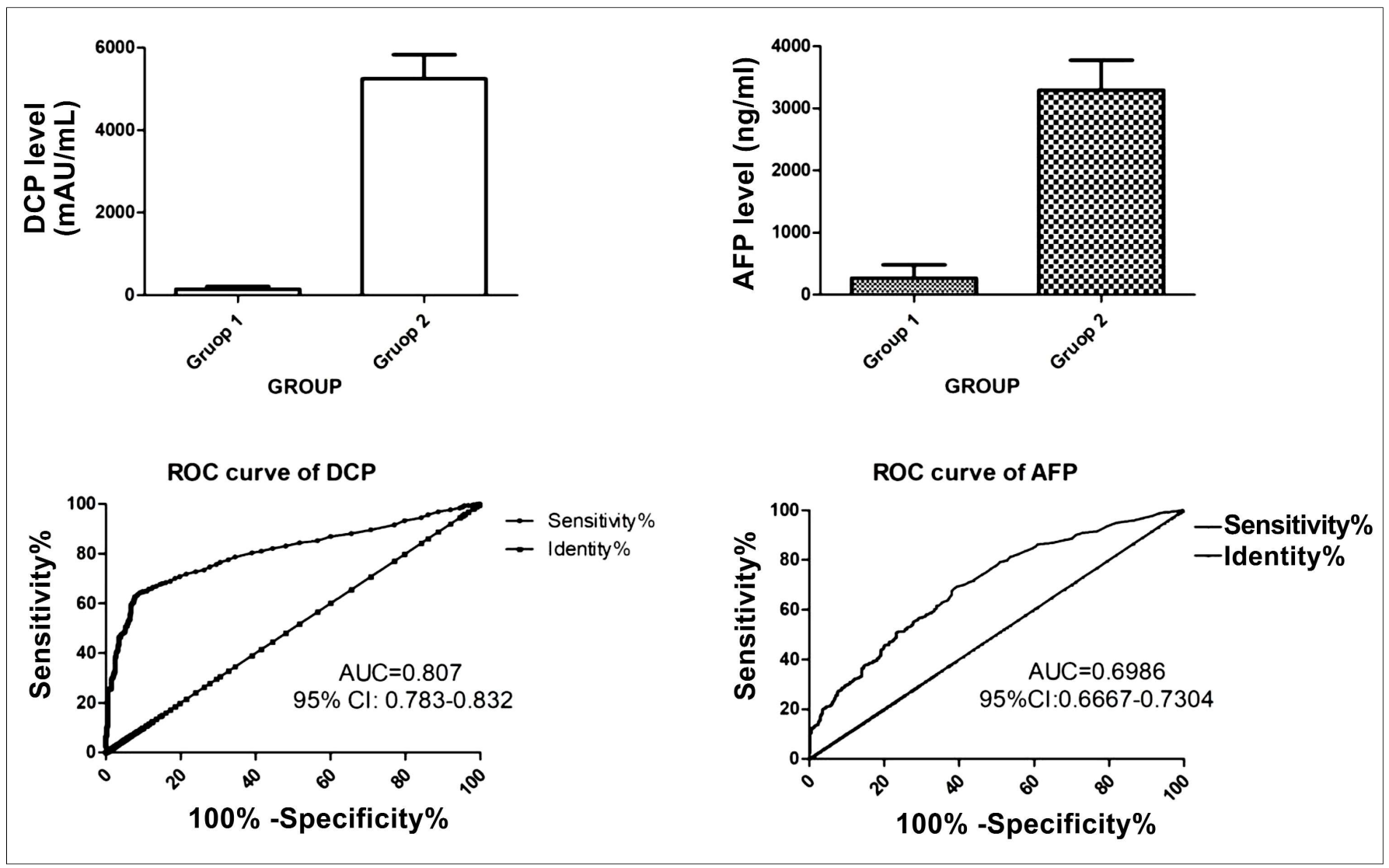

Figure 1: The serum levels and ROC curves of DCP and AFP in Group 1 and Group 2.

Group 1: Non-cancer patients; Group 2: Primary cancer in liver; Sensitivity of DCP: $64.18 \%$; Specificity of DCP: $91 \%$; Positive predictive value of DCP: $93.37 \%$; Negative predictive value of DCP: $56.26 \%$. 
The serum levels of DCP were measured by chemiluminescent enzyme immunoassay (Lumipulse ${ }^{\circledR}$ G PIVKAII; FUJIREBIO INC., Japan) with the instrument of automatic immunoassay analyzer (LUMIPULSE G1200). Serum samples were stored at $-80^{\circ} \mathrm{C}$ until further testing. All processes were preformed according to the manufacturer's instructions. The measuring range for DCP was $0-75000 \mathrm{mAU} / \mathrm{mL}$.

All statistical analyses were accomplished using GraphPad Prism 5, and $\mathrm{p}<0.05$ was considered significant. A receiver operating characteristic (ROC) curve analysis was used to assess the diagnostic accuracy of DCP for primary cancer in liver. Cutoff values and the area under the receiver operating characteristic curve (AUROC) were calculated according to the ROC curve.

\section{RESULTS}

The serum DCP and alpha fetoprotein (AFP) levels in Group 1 and Group 2 were measured and compared. As shown in Figure 1, the levels of both DCP and AFP in the primary cancer in liver group were obviously elevated than the non-cancer patients $(p<0.001)$. To determine the diagnostic efficacy of DCP in primary cancer in liver, ROC curves were plotted according to the measured levels of DCP and AFP (Figure 1). For primary cancer in liver, the best cutoff value was $56.5 \mathrm{mAU} / \mathrm{mL}$. The sensitivity, specificity, positive predictive value and negative predictive value were $64.2 \%, 91 \%, 93.37 \%$ and $56.25 \%$, respectively. The AUROC was $0.807(95 \% \mathrm{Cl}$, 0.783-0.832).

To determine the diagnostic efficacy of DCP in different types of primary cancer in liver (HCC, ICC and cHCC-

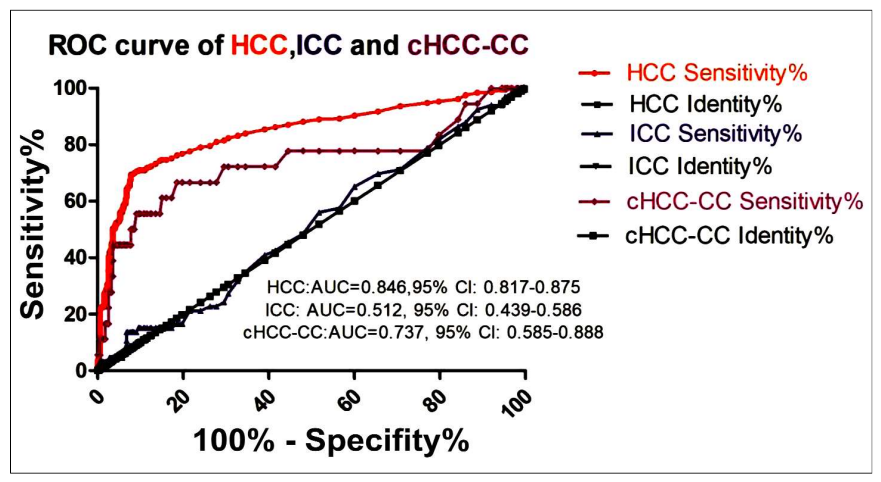

Figure 2: The ROC curve of DCP in HCC, ICC and CHCC-CC patients. For HCC:

Sensitivity of DCP: $69.34 \%$,

Specificity of DCP: $92.25 \%$

Positive predictive value of DCP: $88.97 \%$

Negative predictive value of DCP: $76.88 \%$

For ICC:

Sensitivity of DCP: $13.64 \%$,

Specificity of DCP: $93.25 \%$

Positive predictive value of DCP: $25 \%$

Negative predictive value of DCP: $86.74 \%$

For $\mathrm{CHCC}-\mathrm{CC}$ :

Sensitivity of DCP: $66.67 \%$

Specificity of DCP: $81.5 \%$

Positive predictive value of DCP: $13.95 \%$

Negative predictive value of DCP: $98.19 \%$
CC), ROC curves were plotted according to the measured levels of DCP (Figure 2). For HCC, the sensitivity, specificity, cutoff value, and AUROC of DCP were $69.3 \%, 92.2 \%, 60.5 \mathrm{mAU} / \mathrm{mL}$, and $0.846(95 \% \mathrm{Cl}$ : $0.817-0.875)$, respectively. For ICC, the sensitivity, specificity, cutoff value, and AUROC of DCP were $13.6 \%, 93.2 \%, 76.5 \mathrm{mAU} / \mathrm{mL}$, and $0.512(95 \% \mathrm{Cl}$ : $0.439-0.586)$, respectively. For CHCC-CC, the sensitivity, specificity, cutoff value, and AUROC of DCP were $66.7 \%, 81.5 \%, 40.5 \mathrm{mAU} / \mathrm{mL}$, and $0.737(95 \% \mathrm{Cl}$ : $0.585-0.888)$, respectively.

\section{DISCUSSION}

Primary cancer in liver is a kind of malignancy with a high and continuously rising mortality.3,12 Early diagnosis is extremely important for better prognosis. However, innocuous and easily accessible serum markers that would make diagnosis easier are yet clinically unavailable. In the present study, patients with primary cancer in liver exhibited markedly higher levels of serum DCP than the non-cancer patients and DCP shows excellent diagnostic accuracy in hepatocyte-derived malignant tumors.

The average year of primary cancer in liver patients was 58.18 years old and most of them were males (male vs. female: 665 vs. 125). AFP was widely used as a serum maker for primary cancer in liver but approximately $30 \%$ of liver cancer patients were with a normal AFP. 13 In this study, with both the $p$-value of $<0.001$, the diagnostic efficacy of DCP was much better than AFP. The AUROC, which ranges from 0.5 for accuracy of chance to 1 for perfect accuracy, is the most frequently used method for measuring diagnostic accuracy. ${ }^{14}$ The AUROC of DCP in primary cancer in liver patients of this study was up to 0.807 , while the AUROC of AFP was only 0.6986.

For different histological types, DCP showed rather different degrees of diagnostic accuracies. DCP showed the best diagnostic accuracy in HCC, with an AUROC of 0.846 , higher than the overall AUROC for primary cancer in liver. The diagnostic accuracy of DCP was studied and proven efficient mostly in HCC patients in previous studies, 15,16 but the sensitivity, specificity, and cutoff points in previous studies have been inconsistent, ${ }^{15,17}$ and even conflicting in some cases. 10

This disparity is likely because of two reasons. The first is the differences in diagnostic criteria and the fact that diagnostic values were mainly derived from patients without homogeneous diagnostic criteria. Most diagnoses in previous studies were based on histological examination or imaging characteristics. Histological examination is usually considered the gold standard with the highest diagnostic sensitivity and specificity, and imaging characteristics may result in false positive and false negative cases, especially in early or poorly differentiated infiltrative cancers. ${ }^{18}$ 
The second reason for the disparity could be the different points of cutoff value. The diagnostic technique was revised in 1997 resulting in a much higher sensitivity. ${ }^{19}$ The $40 \mathrm{mAU} / \mathrm{mL}$ cutoff point for DCP as per the manufacturer's instruction, was varified and consulted for an article published in 1996. Most of the previous studies used this $40 \mathrm{mAU} / \mathrm{mL}$ cutoff point directly instead of calculating it according to the measured levels. 15,16,20-22 Review of previous studies on HCC showed that the summary estimates of DCP were as follows: sensitivity, $63 \%(95 \% \mathrm{Cl}$ : $58 \%-67 \%)$ and specificity, $91 \%$ (95\% Cl: $88 \%-93 \%)$ The AUROC was 0.84 for DCP. 16 In this study, about HCC, with the more precise diagnostic criteria (histological examination only) and by employing a calculated cutoff value, the AUROC, sensitivity, and specificity were $0.846,69.3 \%$, and $92.2 \%$, respectively.

cHCC-CC is a kind of primary liver cancer with a more aggressive behaviour and a poorer prognosis than either HCC or ICC. ${ }^{2}$ Accurate preoperative diagnosis of $\mathrm{cHCC}-\mathrm{CC}$ is extremely important because the characteristics of lymph node metastasis can directly influence the outcome of surgery. ${ }^{2}$ However, neither specific imaging characteristics nor suitable serum markers have been discovered for CHCC-CC. 9 In this study, the authors first studied the diagnostic accuracy of DCP among CHCC-CC patients (AUROC: $0.737,95 \% \mathrm{Cl}$ : $0.585-0.888$ ). The sensitivity and specificity were $66.7 \%$ and $81.5 \%$, respectively. ICC has distinct pathogenic mechanisms, molecular pathways, and biological characteristics from HCC. ${ }^{23}$ In the cases of ICC, AUROC was only 0.512 .

As shown in this study, DCP is a useful serum marker in primary cancer in liver and a specific biomarker for hepatocyte-derived malignant tumors. It shows excellent diagnostic accuracy in both HCC and CHCC-CC, but limited diagnostic accuracy in ICC. In primary cancer in liver and its different histological types, it is a useful biomarker not only for diagnosis but also for differential diagnosis. However, DCP has not been widely employed worldwide, ${ }^{24}$ especially among the high incidence areas such as China and western Africa. ${ }^{12}$

Serum DCP levels will likely be introduced into mainstream clinical testing only when there is increased research and a better understanding of the topic. Meanwhile, although DCP has been studied for nearly 40 years and its diagnostic accuracy has been widely verified, the exact metabolism of DCP has not been fully elucidated. It is possibly because of decreased activity of the gamma-glutamyl carboxylase enzyme or altered vitamin $\mathrm{K}$ metabolism. ${ }^{25}$ Similarly, the pathogenesis of $\mathrm{HCC}$ and $\mathrm{CHCC}-\mathrm{CC}$ are still unclear. Considering the excellent diagnostic accuracy of DCP in hepatocytederived malignant tumors, the pathogenesis or therapeutic target may be derived from DCP. The retrospective design and limited number of $\mathrm{CHCC}-\mathrm{CC}$ patients are limitations of this study. A prospective study with more samples of $\mathrm{CHCC}-\mathrm{CC}$ is need in the future.

\section{CONCLUSION}

The analysis of the present study suggested that DCP is an effective marker for differentiating between $\mathrm{HCC}$ and cHCC-CC. DCP could be considered not only for surveillance and diagnosis of primary cancer in liver, but also for differential diagnosis of its different histological types.

\section{ETHICAL APPROVAL:}

The study was approved prior to initiation of the research work by the Ethics Committees of the First Affiliated Hospital, College of Medicine, Zhejiang University.

\section{PATIENTS' CONSENT:}

As a retrospective cohort study, a waiver for informed consent was obtained, and all the data from patients were analysed anonymously.

\section{CONFLICT OF INTEREST:}

Authors declared no conflict of interest.

\section{AUTHORS' CONTRIBUTION:}

YL: Responsible for the conception and design of the work, revising it critically for important intellectual content.

JC: Responsible for the analysis of data for the work, final approval of the version to be published and agreement to be accountable for all aspects of the work in ensuring that questions related to the accuracy or integrity of any part of the work are appropriately investigated and resolved.

\section{REFERENCES}

1. Petrick JL, Braunlin M, Laversanne M, Valery PC, Bray F, McGlynn KA. International trends in liver cancer incidence, overall and by histologic subtype, 1978-2007. Int J Cancer 2016; 139:1534-45.

2. O'Connor K, Walsh JC, Schaeffer DF. Combined hepatocellular-cholangiocarcinoma (cHCC-CC): A distinct entity. Ann Hepatol 2014; 13:317-22.

3. Burkhart RA, Pawlik TM. Staging and prognostic models for hepatocellular carcinoma and intrahepatic cholangiocarcinoma. Cancer Control 2017; 24:1073274817729235.

4. Hou SC, Xiao MB, Ni RZ, Ni WK, Jiang F, Li XY, et al. Serum GP73 is complementary to AFP and GGT-II for the diagnosis of hepatocellular carcinoma. Oncol Lett 2013; 6:1152-8.

5. Malaguarnera G, Paladina I, Giordano M, Malaguarnera M, Bertino G, Berretta M. Serum markers of intrahepatic cholangiocarcinoma. Dis Markers 2013; 34:219-28.

6. Llovet JM, Ducreux M, Lencioni R, Di Bisceglie AM, Galle PR, Dufour JF, et al. EASL-EORTC clinical practice guidelines: Management of hepatocellular carcinoma. J Hepatol 2012; 56: 908-43.

7. Hanna RF, Miloushev VZ, Tang A, Finklestone LA, Brejt SZ, Sandhu RS, et al. Comparative 13-year meta-analysis of the 
sensitivity and positive predictive value of ultrasound, CT, and MRI for detecting hepatocellular carcinoma. Abdom Radiol (NY) 2016; 41:71-90.

8. Chen LD, Xu HX, Xie XY, Xie XH, Xu ZF, Liu GJ, et al. Intrahepatic cholangiocarcinoma and hepatocellular carcinoma: Differential diagnosis with contrast-enhanced ultrasound. Eur Radiol 2010; 20:743-53.

9. Maximin S, Ganeshan DM, Shanbhogue AK, Dighe MK, Yeh MM, Kolokythas $\mathrm{O}$, et al. Current update on combined hepatocellular-cholangiocarcinoma. Eur J Radiol Open 2014; 1:40-8.

10. Li GJ, Chen QY, Harrison TJ, Wang XY, Hu LP, Yang QL, et al. Des-gamma carboxyprothrombin may not be a good biomarker for hepatocellular carcinoma in those chronically infected with hepatitis $B$ virus with basal core promoter double mutations $\left(T^{\wedge}\{1762\}, A^{\wedge}\{1764\}\right)$, a prospective study. Cancer Biomark 2017; 18:241-8.

11. Qin S. Guidelines on the diagnosis and treatment of primary liver cancer (2011 edition). Chin Clin Oncol 2012; 1:10.

12. Ferlay J, Soerjomataram I, Dikshit R, Eser S, Mathers C, Rebelo $\mathrm{M}$, et al. Cancer incidence and mortality worldwide: Sources, methods and major patterns in GLOBOCAN 2012. Int J Cancer 2015; 136:E359-86.

13. Zhou J, Sun HC, Wang Z, Cong WM, Wang JH, Zeng MS, et al. Guidelines for diagnosis and treatment of primary liver cancer in China (2017 Edition). Liver Cancer 2018; 7:235-60.

14. Alemayehu D, Zou KH. Applications of ROC analysis in medical research: Recent developments and future directions. Acad Radiol 2012; 19:1457-64.

15. Marrero JA, Feng Z, Wang Y, Nguyen MH, Befeler AS, Roberts LR, et al. Alpha-fetoprotein, des-gamma carboxyprothrombin, and lectin-bound alpha-fetoprotein in early hepatocellular carcinoma. Gastroenterology 2009; 137:110-8.

16. Li C, Zhang Z, Zhang P, Liu J. Diagnostic accuracy of desgamma-carboxy prothrombin versus alpha-fetoprotein for hepatocellular carcinoma: A systematic review. Hepatol Res 2014; 44:E11-25.
17. Lok AS, Sterling RK, Everhart JE, Wright EC, Hoefs JC, Di Bisceglie AM, et al. Des-gamma-carboxy prothrombin and alpha-fetoprotein as biomarkers for the early detection of hepatocellular carcinoma. Gastroenterology 2010; 138:493-502.

18. Hennedige T, Venkatesh SK. Advances in computed tomography and magnetic resonance imaging of hepatocellular carcinoma. World J Gastroenterol 2016; 22:205-20.

19. Gao P, Li M, Tian QB, Liu DW. Diagnostic performance of desgamma-carboxy prothrombin (DCP) for hepatocellular carcinoma: A bivariate meta-analysis. Neoplasma 2012; 59: 150-9.

20. Sterling RK, Jeffers L, Gordon F, Venook AP, Reddy KR, Satomura $S$, et al. Utility of lens culinaris agglutinin-reactive fraction of alpha-fetoprotein and des-gamma-carboxy prothrombin, alone or in combination, as biomarkers for hepatocellular carcinoma. Clin Gastroenterol Hepatol 2009; 7:104-13.

21. Gao J, Feng X, Inagaki Y, Song P, Kokudo N, Hasegawa K, et al. Des-gamma-carboxy prothrombin and c-Met were concurrently and extensively expressed in hepatocellular carcinoma and associated with tumor recurrence. Biosci Trends 2012; 6:153-9.

22. Tateishi R, Yoshida H, Matsuyama Y, Mine N, Kondo Y, Omata M. Diagnostic accuracy of tumor markers for hepatocellular carcinoma: A systematic review. Hepatol Int 2008; 2:17-30.

23. Cong WM, Bakker A, Swalsky PA, Raja S, Woods J, Thomas S, et al. Multiple genetic alterations involved in the tumorigenesis of human cholangiocarcinoma: A molecular genetic and clinicopathological study. J Cancer Res Clin Oncol 2001; 127:187-92.

24. Song P, Gao J, Inagaki Y, Kokudo N, Hasegawa K, Sugawara Y, et al. Biomarkers: Evaluation of screening for and early diagnosis of hepatocellular carcinoma in Japan and China. Liver Cancer 2013; 2:31-9.

25. Inagaki Y, Tang W, Makuuchi M, Hasegawa K, Sugawara Y, Kokudo N. Clinical and molecular insights into the hepatocellular carcinoma tumour marker des-gamma-carboxyprothrombin. Liver Int 2011; 31:22-35. 